INFLAMMATORY BOWEL DISEASE

\title{
Local delivery of adenoviral vectors encoding murine interleukin 10 induces colonic interleukin 10 production and is therapeutic for murine colitis
}

\author{
J O Lindsay, C J Ciesielski, T Scheinin, F M Brennan, H J Hodgson
}

Gut 2003;52:981-987

\begin{abstract}
Introduction: Interleukin 10 knockout (IL-10-/-) mice spontaneously develop a Th1 T cell mediated colitis with many similarities to Crohn's disease. Daily injections of IL- 10 are unable to induce remission in mice with established disease. In contrast, we have shown previously that intravenous administration of adenoviral vectors encoding IL-10 (AdvmulL-10) induces hepatic IL-10 release and leads to long term disease suppression with profound systemic immunoregulatory changes.

Aims: To determine whether rectal delivery of AdvmulL- 10 induces localised colonic IL- 10 expression without systemic immune suppression, and assess its therapeutic efficacy in IL-10-/- mice with established colitis.

Results: A single rectal infusion of $5 \times 10^{8}$ PFU AdvmulL- 10 to 10 week IL- $10-/-$ mice resulted in a median level of $27.3 \mathrm{pg} / \mathrm{mg} \mathrm{IL-10}$ in colonic homogenates harvested one week later. IL-10-/- mice with established colitis treated with an enema of $5 \times 10^{8}$ PFU AdvmulL- 10 entered clinical and histological remission whereas empty cassette adenovirus (AdvO) or phosphate buffered saline (PBS) treated mice developed progressive disease. After four weeks, the histological score of AdvmulL-10 treated mice (4.4 (1.5)) was significantly lower than that of AdvO (1 1.1 (1.1); $p<0.001$ ) and PBS (10.9 (1.0); $p<0.01)$ treated controls. In addition, the stool concentration of IL-1 $\beta$ over the four week experiment was significantly higher in mice treated with saline or AdvO than in those treated with AdvmulL-10 $(p<0.01)$.

Conclusion: Local AdvmulL-10 therapy reverses colitis in IL-10-/- mice without the systemic effects seen after intravenous administration. Gene therapy strategies using adenoviral vectors encoding immunoregulatory cytokines may prove to be a potent approach to the treatment of chronic inflammatory diseases such as Crohn's disease.
\end{abstract}

See end of article for authors' affiliations

\section{Correspondence to:} Dr J O Lindsay, Kennedy Institute of Rheumatology Division, Imperial College School of Medicine, 1 Aspenlea Road, London W6 8LH, UK;

i.lindsay@doctors.org.uk

Accepted for publication 18 September 2002
$\mathrm{T}$ he chronic gastrointestinal inflammation that characterises Crohn's disease is associated with loss of tolerance to resident enteric bacteria, ${ }^{1}$ activation of immune cells, and leucocytic infiltration. ${ }^{2}$ These inflammatory processes are orchestrated by the production of chemokines, proinflammatory monokines, and Thl cytokines. ${ }^{3}$ Studies of mice in which interleukin (IL)-10 signalling is disrupted provide compelling evidence for the role of this immunoregulatory cytokine in maintaining mucosal homeostasis. Thus both gene targeted IL10 knockout (IL-10-/-) and IL-10 receptor 2 deficient mice develop a Thl lymphocyte mediated colitis with many similarities to Crohn's disease. ${ }^{45}$ IL-10 has broad immunoregulatory activity and thus acts to suppress intestinal inflammation on several levels. ${ }^{6}$ It diminishes both antigen presentation and IL-12 release, which inhibits differentiation of Thl lymphocytes. In addition, IL-10 suppresses the release of many other proinflammatory cytokines and chemokines, including tumour necrosis factor $\alpha$ (TNF- $\alpha$ ), IL- 1, IL-6, and IL-8. Finally, there is strong evidence that IL-10 acts to promote differentiation and augment the activity of regulatory $\mathrm{T}$ cells. ${ }^{7}$

Administration of systemic IL-10 injections is sufficient to inhibit proinflammatory Thl lymphocyte responses and abrogate colitis in both dextran sodium sulphate and $\mathrm{CD}^{+} \mathrm{CD} 4 \mathrm{RB}^{\text {high }}$ transfer models of colitis. ${ }^{8}{ }^{9}$ Furthermore, in vitro studies have shown that exogenous IL-10 downregulates enhanced proinflammatory cytokine release from lamina propria mononuclear cells isolated from patients with Crohn's disease. ${ }^{10}$ Despite this, two recent clinical trials in patients with either steroid naïve or steroid resistant Crohn's disease have shown that daily systemic IL-10 injections are safe and well tolerated but have minimal therapeutic efficacy compared with placebo. ${ }^{11}{ }^{12}$ One explanation for these disappointing results is that the pharmacodynamics of daily systemic IL-10 delivery does not allow sufficient mucosal penetration to inhibit the proinflammatory response. Thus strategies that result in a more sustained and focused delivery of IL-10 to the gastrointestinal mucosa may prove effective.

Recently, we have demonstrated that a single systemic injection of an adenoviral vector encoding murine IL-10 (AdvmuIL-10) targets hepatocytes and results in at least 10 weeks of bioactive IL-10 expression. ${ }^{13}$ Unlike daily recombinant IL-10 injections, ${ }^{14}$ systemic AdvmuIL-10 therapy induced remission in IL-10-/- mice with established disease, leading to lower clinical and histological scores with reduced faecal proinflammatory cytokine levels. Finally, delivery of IL-10 also appeared to limit the host response to viral vector, as antiadenovirus antibody titres were lower in AdvmuIL-10 treated mice than in littermates treated with control virus. However, in addition to these beneficial effects, systemic AdvmuIL-10 therapy led to generalised immunoregulatory changes in IL-10-/- mice, such as an impaired splenocyte response to lipopolysaccharide (LPS), that may limit its potential in patients with Crohn's disease. Likewise, clinical

Abbreviations: IL-10, interleukin 10; AdvmulL-10, adenoviral vector encoding murine IL- 10; Advßgal, adenoviral vector encoding $\beta$-galactosidase; Adv0, empty cassette adenoviral vector; TNF- $\alpha$, tumour necrosis factor $\alpha$; IFN- $\gamma$, interferon $\gamma$; LPS, lipopolysaccharide; FCS, fetal calf serum; PBS, phosphate buffered saline; DMEM, Dulbecco's modified Eagle's medium; CAR, Coxsackie virus and adenovirus receptor; LPMN, lamina propria mononuclear cell; $\mathrm{MOI}$, multiplicity of infection. 
trials of systemic IL-10 have reported a dose dependant but fully reversible anaemia and thrombocytopenia and the long term sequelae of this treatment are not known. ${ }^{11}{ }^{12}$

It is possible that targeted local IL-10 would be sufficient to treat intestinal inflammation while avoiding the side effects associated with systemic therapy. Thus oral administration of non-pathogenic bacteria (Lactococcus lactis) that have been genetically modified to secrete murine IL-10 led to histological improvement in dextran sodium sulphate and IL-10-/- models of colitis. ${ }^{15}$ Concerns raised by the release of genetically modified organisms into the environment may be avoided by the use of replication deficient adenoviral vectors such as AdvmuIL-10. Adenoviruses have strong tropism for epithelial tissues, and adenoviral vectors delivered per rectum have been shown to induce expression of the delivered transgene within colonocytes. ${ }^{16}$ In this paper, we demonstrate the ability of rectal AdvmuIL-10 to induce colonic IL-10 expression and ameliorate established colitis in IL-10-/- mice without the generalised side effects associated with systemic therapy. Furthermore, we show that local AdvmuIL-10 results in a diminished host antiadenoviral response compared with control adenoviral vectors.

\section{MATERIALS AND METHODS}

General reagents were of research grade and purchased from Sigma (St Louis, Missouri, USA). All reagents used for cell culture were determined to be LPS free using a limulus amoebocyte lysate assay (Biowhitacker, Berkshire, UK), as directed by the manufacturer ( sensitivity $<10 \mathrm{pg} / \mathrm{ml}$ ).

\section{Adenoviral vectors}

The recombinant El deleted type 5 adenoviral vectors, encoding murine IL-10 under the transcriptional control of the rous sarcoma virus promoter (AdvmuIL-10), $\beta$-galactosidase under the cytomegalovirus promoter (Adv $\beta \mathrm{Gal})$, or having no insert (Adv0), were generously donated by Professor Dallman (Imperial College, London, UK). Viruses were propagated in the 293 human embryonic kidney cell line (Quantum Biotechnology Inc., Canada) and purified by ultracentrifugation through two caesium chloride gradients (Boehringer Mannheim, Lewes, Sussex, UK). The titre of adenoviral vectors was determined by plaque assay on 293 cells. Viral stocks were diluted with 10\% glycerol and stored in aliquots at $-80^{\circ} \mathrm{C}$ until use. ${ }^{17}$

\section{In vitro epithelial cell infection}

The transformed human colonic epithelial cell lines HT29 and SW620 (ATCC, Maryland, USA) were cultured at a density of $1 \times 10^{6} / \mathrm{ml}$ in RPMI 1640 medium (PAA Laboratories Ltd, Yeovil, UK) supplemented with $10 \%$ fetal calf serum (FCS), $100 \mathrm{u} / \mathrm{ml}$ penicillin, and $100 \mu \mathrm{g} / \mathrm{ml}$ streptomycin (Biowhitacker). Initial experiments demonstrated that at least 95\% infection with Advßgal was obtained with a multiplicity of infection of (MOI) 50:1 and an incubation time of 36 hours; thus these conditions were used in subsequent experiments. Cells were cultured in triplicate, infected with Adv0, AdvmuIL-10, or saline vehicle and cultured for 28 days with weekly passaging. Supernatants were sampled daily and frozen until assay. IL-10 bioactivity was determined by the ability of serial dilutions of the supernatant to inhibit TNF- $\alpha$ release from a murine monocyte cell line (RAW cells; ATCC) plated at $1 \times 10^{5} / \mathrm{ml}$ in a 96 well plate stimulated with $10 \mathrm{ng} / \mathrm{ml}$ LPS. Serial dilutions of recombinant murine IL-10 was used as a standard while the specificity of the effect was determined by preincubation of the supernatants and standards with $10 \mu \mathrm{g} / \mathrm{ml}$ of a rat monoclonal antimurine IL-10 antibody (JES52A5; donated by DNAX Research Institute, California, USA) or a rat IgG1 isotype control (OX20; ATCC).

\section{Animals}

IL-10-/- mice on a C57BL/6 background (purchased from Harlan UK Ltd, Oxon, UK) were backcrossed for one generation onto DBA/l mice to increase breeding vigour and disease expression. Progeny from the interbreeding of the heterozygous offspring were used in all experiments. Newly bred mice were screened for the homozygote IL-10-/- or wildtype genotype (C57BL/6xDBAl), as described previously. ${ }^{13}$ Mice were maintained in specific pathogen free conditions in a laminar flow hood at all times with free access to food and water. All experiments involving animals were approved by the local ethics review process committee and performed under license from the Home Office.

Preliminary experiments demonstrated that under standard laboratory conditions, IL-10-/- animals developed a progressive colitis from four weeks of age. Clinical manifestations of disease included the passage of mucous, diarrhoea, rectal prolapse, and weight loss of greater than $5 \%$ of total body weight. Mice were examined weekly, and given a clinical score that consisted of one point for each of the above signs. Previous work has demonstrated a close correlation between the clinical score and histological severity of colitis (correlation coefficient 0.865 ; Dr T Scheinin, personal communication).

\section{Experimental protocols}

Ten week old IL-10-/- mice with established colitis received $5 \times 10^{8}$ PFU of AdvmuIL-10, Adv0, or phosphate buffered saline (PBS) vehicle as a $100 \mu \mathrm{l}$ rectal infusion under sedation. Mice were held vertically for one minute and then suspended on tilted racks for a further 10 minutes to prevent seepage. Wildtype C57BL/6xDBAl mice were used as a negative control. The clinical score of each animal was assessed and stool samples were collected weekly throughout the experiment. Animals were sacrificed by cervical dislocation four weeks after adenoviral therapy. Serum was collected via cardiac puncture, spleens were harvested, stool samples collected, and serial segments of colon, caecum, and ileum were fixed in 10\% neutral buffered formalin for histological analysis. In a separate experiment, IL-10-/- mice that had received saline or AdvmuIL-10 by rectal instillation were sacrificed after seven days. The liver, spleen, and colon of these animals were weighed and homogenised in $5 \mu \mathrm{l}$ PBS per mg tissue. Supernatants were harvested after centrifugation and stored at $-20^{\circ} \mathrm{C}$ until assay.

\section{Histological analysis}

Samples were routinely processed, embedded in paraffin, sectioned at $5 \mu \mathrm{m}$, and stained with haematoxylin and eosin for light microscopic examination. Histological assessment was performed by an investigator (TS) blinded to the treatment group. The terminal ileum and five segments of colon were examined per mouse and each given a histological score from 0 to 4 as described previously ${ }^{14}$; thus a total score for each mouse from 0 (no change in any segment) to 24 (grade 4 changes in all segments) was obtained. Scores of less than 5 were deemed to be within normal limits.

\section{Stool samples}

Stool samples were collected weekly from all animals and weighed. Samples were emulsified in $500 \mu \mathrm{l}$ per $100 \mu \mathrm{g}$ stool weight of a solution of $1 \mathrm{mg} / \mathrm{ml}$ soy trypsin inhibitor and $1 \mathrm{mg} / \mathrm{ml}$ phenylmethylsulphonyl fluoride in PBS. Supernatants were collected after centrifugation at $10000 \mathrm{~g}$ for 15 minutes and stored at $-20^{\circ} \mathrm{C}$.

\section{Spleen cell cultures}

After sacrifice, each spleen was placed in RPMI 1640 supplemented with $10 \%$ FCS, $100 \mathrm{u} / \mathrm{ml}$ penicillin, and 100 $\mu \mathrm{g} / \mathrm{ml}$ streptomycin. Cell suspensions were obtained by passing tissue through a $200 \mu \mathrm{m}^{2}$ nylon mesh. After erythrocyte lysis, cells were washed in Hanks balanced salt solution three times before resuspension in medium. Cells were plated at $2 \times 10^{6} /$ well in 12 well plates (Falcon, Becton Dickinson Labware, USA) in a final volume of $1 \mathrm{ml}$ of medium 

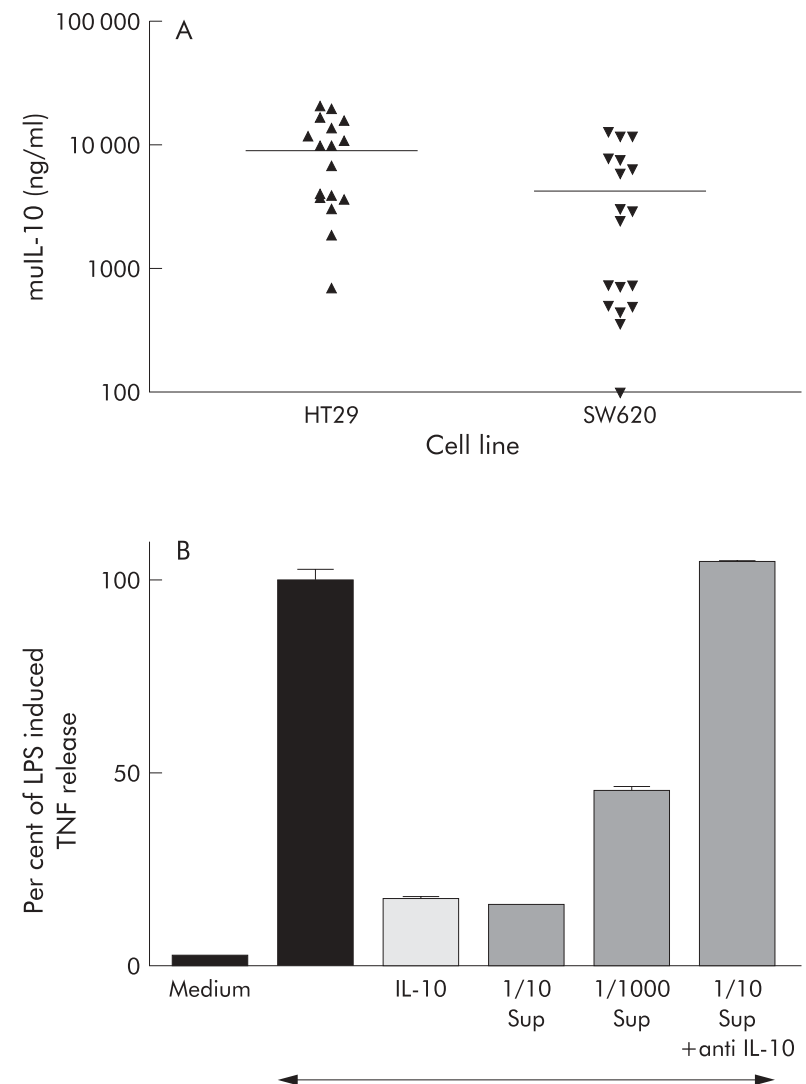

LPS $10 \mathrm{ng} / \mathrm{ml}$

Figure 1 Adenoviral vector encoding murine interleukin 10 (AdvmulL-10) induced bioactive IL-10 release from epithelial cells in vitro. HT29 and SW620 cells were plated at $1 \times 10^{6} / \mathrm{ml}$ in a 12 well plate and infected with Advmull- 10 or empty cassette adenoviral vector (AdvO, MOI 50:1). Supernatants were sampled at 36 hours and daily thereafter. Cultures were passaged 1:2 every week. No IL- 10 was detected in Adv0 infected epithelial cells. (A) IL- 10 release from AdvmulL-10 infected cells. (B) RAW cells $\left(2 \times 10^{5}\right)$ were plated onto a 96 well plate and incubated with IL- $1010 \mathrm{ng} / \mathrm{ml}$, dilutions of the supernatant (Sup) from Advmull-10 infected HT29 cells, or supernatant that had been preincubated with $10 \mu \mathrm{g} / \mathrm{ml}$ (final) antimurine IL-10 antibody for one hour. Cells were cultured for 24 hours with or without $10 \mathrm{ng} / \mathrm{ml}$ lipopolysaccharide (LPS). Supernatants were harvested and assayed for tumour necrosis factor $\alpha(T N F-\alpha)$ release by ELISA. Results are expressed as a percentage of the LPS induced TNF- $\alpha$ response (mean of three experiments). Identical results were obtained using supernatants from SW620 cells.

with or without LPS $10 \mu \mathrm{g} / \mathrm{ml}$, recombinant muIL-10 $10 \mathrm{ng} / \mathrm{ml}$ (Schering Plough, New Jersey, USA), $10 \mu \mathrm{g} / \mathrm{ml}$ neutralising rat antimurine IL-10 antibody (JES52A5; donated by DNAX Research Institute), or a rat IgGl isotype control (OX20; ATCC). Cultures were maintained for 24 hours before supernatants were harvested and stored at $-20^{\circ} \mathrm{C}$.

\section{Measurement of serum antiadenoviral antibody response}

The neutralising antiadenovirus antibody response was analysed in serum from untreated or treated IL-10-/- mice four weeks after gene transfer with $5 \times 10^{8} \mathrm{PFU}$ of AdvmuIL-10, Adv0, or PBS vehicle. ${ }^{18}$ Serum samples $(100 \mu \mathrm{l})$ were heat inactivated at $56^{\circ} \mathrm{C}$ for 30 minutes and diluted twofold in serum free Dulbecco's modified Eagle's medium (DMEM). Each dilution was incubated for 90 minutes at $37^{\circ} \mathrm{C}$ with $2 \times 10^{\circ}$ PFU of Advßgal, and applied in duplicate to $80 \%$ confluent 293 cells on a 96 well plate. After one hour at $37^{\circ} \mathrm{C}, 50 \mu \mathrm{l}$ of DMEM containing $10 \%$ FCS was added to each well, and cells were cultured for a further 36 hours. Cell supernatants were then removed and replaced with $30 \mu \mathrm{l}$ of $0.25 \mathrm{mM}$ Tris $\mathrm{HCl}$, pH 7.8.
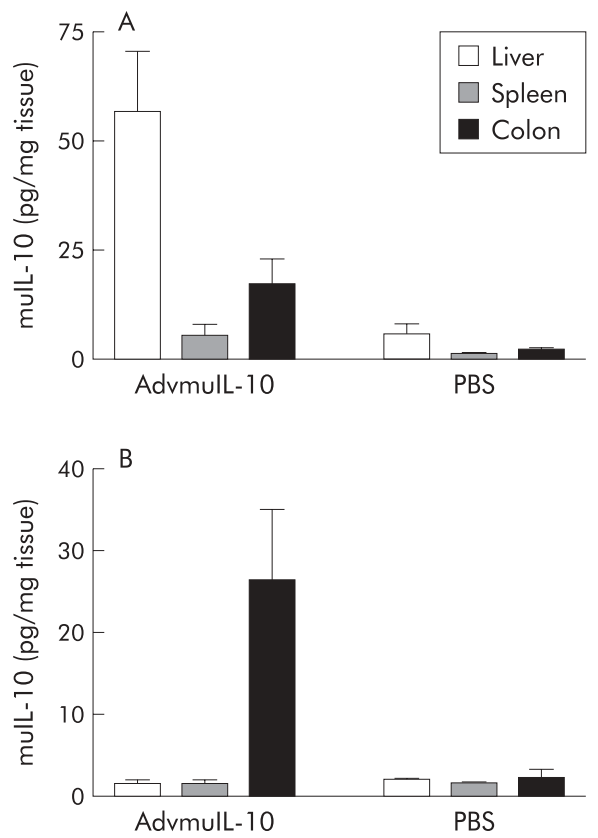

Figure 2 Local adenoviral vector encoding murine interleukin 10 (AdvmulL-10) induced colonic IL- 10 release. Ten week old IL-10-/mice received $5 \times 10^{8}$ PFU of AdvmulL- 10 or saline vehicle either by tail vein injection $(A)$ or rectal infusion $(B)$ under light sedation $(n=3$ /group). After seven days, mice were sacrificed by cervical dislocation. The liver, spleen, and colon were homogenised in $5 \mu \mathrm{l}$ phosphate buffered saline (PBS) per mg tissue. After centrifugation, supernatants were assayed for murine IL- 10 by ELISA (sensitivity 8 $\mathrm{pg} / \mathrm{mg})$.

Plates were vortexed, frozen, and then thawed for three cycles to detach and lyse cells, and centrifuged at $1000 \mathrm{rpm}$ for 20 minutes. The supernatant $(10 \mu \mathrm{l})$ from each well was mixed with $90 \mu \mathrm{l}$ of a $\beta$-galactosidase substrate solution containing $1 \mathrm{mg} / \mathrm{ml} o$ - nitrophenyl- $\beta$-D-galactopyranoside, $1 \mathrm{mM} \mathrm{MgCl}$, $45 \mathrm{mM} 2$-mercaptoethanol, in $0.1 \mathrm{M}$ sodium phosphate buffer, $\mathrm{pH}$ 7.5. The enzyme reaction was stopped after five minutes with addition of $150 \mu \mathrm{l}$ of $0.1 \mathrm{M} \mathrm{Na}_{2} \mathrm{CO}_{3}$, and plates were read at $405 \mathrm{~nm}$. Mean optical density of serum from five animals for each group was compared.

\section{Cytokine analysis}

Cytokine concentrations were measured by sandwich ELISA using paired antibodies according to the manufacturer's recommendations (IL-10 and TNF- $\alpha$ were purchased from Pharmingen, Sorentino, California, USA; interferon $\gamma($ IFN- $\gamma)$ was purchased from Genzyme diagnostics, USA; IL- $1 \beta$ was purchased from R\&D Systems (Abingdon, Oxon, UK)).

\section{Statistical analysis}

Data that exhibited a normal distribution were analysed using a two tailed $t$ test; for comparison of more than two means a two way analysis of variance (ANOVA) was performed.

\section{RESULTS}

In vitro infection of epithelial cell lines with AdvmulL-10 induces bioactive murine IL- 10 release

In order to ascertain whether AdvmuIL-10 induces IL-10 secretion from epithelial cells, cultures of the human colonic epithelial cell lines HT29 and SW620 were infected with AdvmuIL-10 at an MOI of 50:1 for 36 hours; separate wells were infected with Adv0 or saline vehicle as controls. Supernatants were sampled daily, and cultures were passaged weekly. No IL-10 was detected in the supernatants of Adv0 or vehicle treated cells. In contrast, 36 hours after AdvmuIL-10 infection, the supernatants of HT29 and SW620 cells contained 297 (44) ng/ml and 790 (43) 

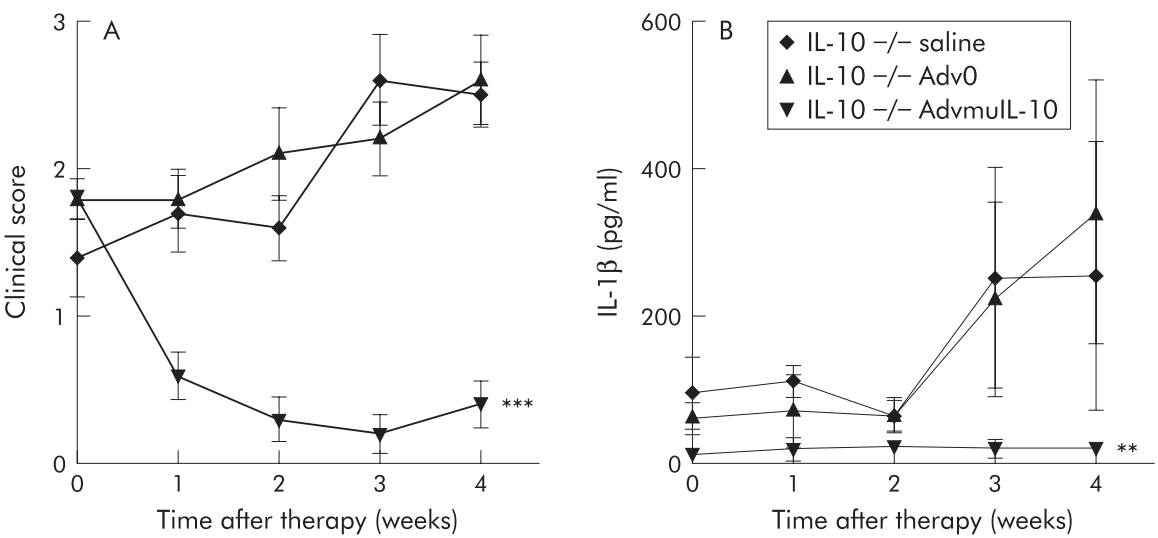

Figure 3 Local adenoviral vector encoding murine interleukin 10 (AdvmulL-10) was therapeutic for established murine colitis. IL-10-/- mice ( $n=10$ /group) with clinical evidence of colitis and C57BL/6 XDBA1 wild-type controls ( $n=5 /$ group) received $5 \times 10^{8}$ PFU of Advmull-10, empty cassette adenoviral vector (AdvO), or vehicle by rectal instillation under light sedation. (A) Clinical score (one point each for rectal mucous, rectal prolapse, diarrhoea, and weight loss $>5 \%$ body weight) and (B) stool IL-1 $\beta$ levels were measured weekly, and are expressed as mean (SEM) for each group. ${ }^{* *} p<0.01,{ }^{* * *} p<0.001$, two way ANOVA.
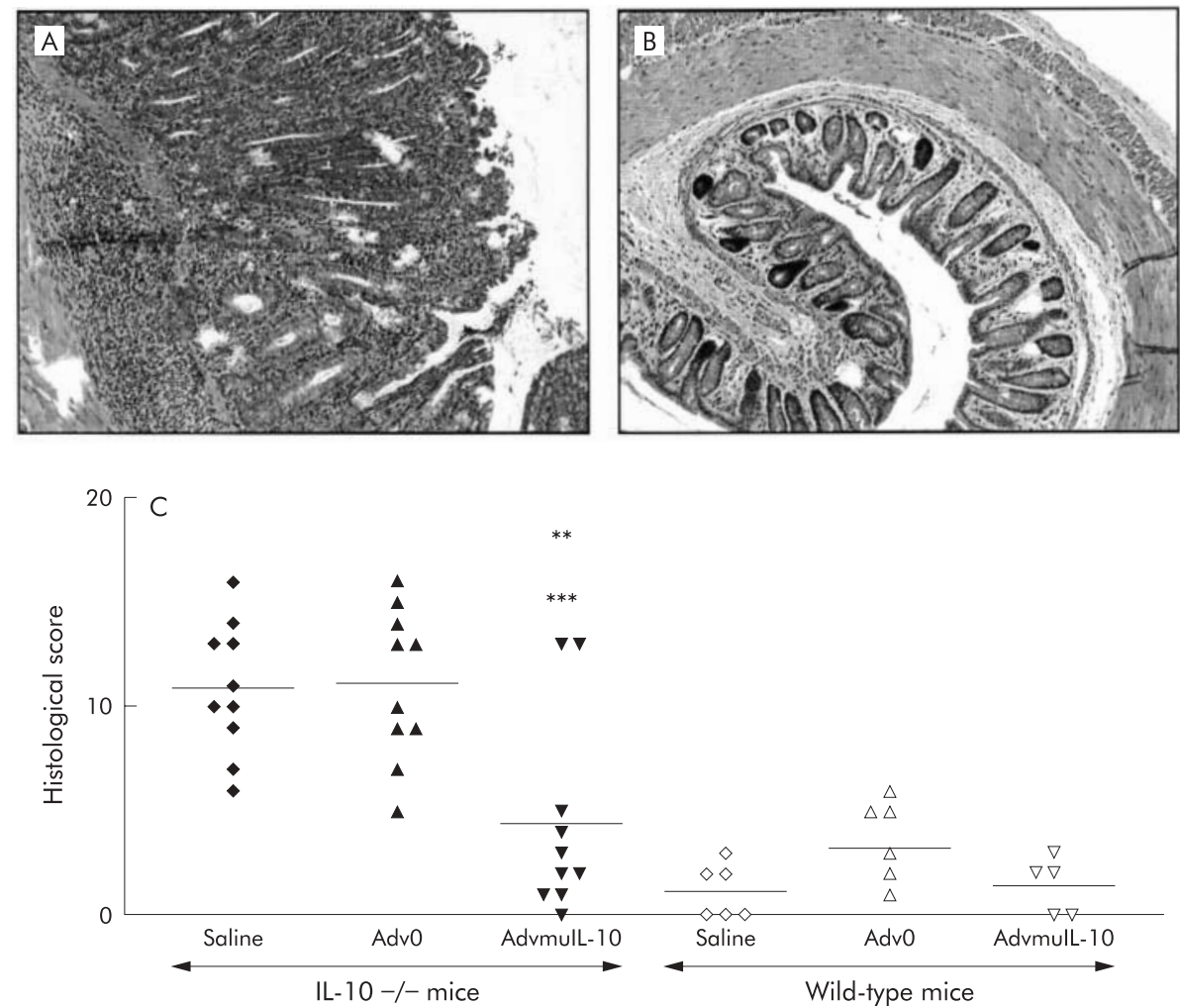

Figure 4 Local adenoviral vector encoding murine interleukin 10 (AdvmulL-10) therapy reduced histological colitis scores in IL-10-/- mice with established disease. Four weeks after therapy, mice were sacrificed by cervical dislocation and sections of five regions of the colon were processed for histology. Representative samples from an IL-10-/- mouse treated with empty cassette adenoviral vector (AdvO) (A) and Advmull-10 (B) are shown. An investigator, blinded to treatment group, gave each section an inflammatory score from 0 to 4 . The sum of these scores is shown for each mouse (C), with the bar representing the mean for the group $\left({ }^{* *} p<0.01\right.$ versus IL- $10-/-$ saline group; ${ }^{* *} p<0.001$ versus IL-10-/- Adv0 group; one way ANOVA with Bonferroni correction).

ng/ml muIL-10, respectively (mean (SEM)). IL-10 release continued for the 28 days of the experiment, as shown in fig 1 . In order to confirm that this IL-10 was bioactive, the ability of the supernatants from AdvmuIL-10 infected HT29 cells to inhibit LPS induced TNF- $\alpha$ release from cultures of RAW cells was compared with recombinant murine IL-10. muIL-10 10 $\mathrm{ng} / \mathrm{ml}$ reduced TNF- $\alpha$ release to $17.5(0.7) \%$ of LPS stimulated levels whereas a $1 / 10$ dilution of the supernatant of AdvmuIL-10 infected HT29 cells reduced TNF- $\alpha$ release to 15.8 $(0.29) \%$. Addition of a monoclonal antimurine IL-10 antibody (but not isotype control antibody) abrogated this inhibitory effect completely (fig 1).

\section{Rectal instillation of AdvmulL-10 results in localised} colonic IL-10 expression

Systemic delivery of adenoviral vectors predominantly targets hepatocytes although some splenic and colonic expression of the delivered transgene has been reported. ${ }^{19}$ In order to assess whether rectal AdvmuIL-10 delivery results in localised colonic IL- 10 release, $5 \times 10^{8}$ PFU of AdvmuIL-10 or PBS vehicle were delivered to 10 week old IL-10-/- mice either by tail vein injection or by rectal instillation ( $\mathrm{n}=3$ /group). Mice were sacrificed one week after adenoviral administration. IL-10 concentration in colonic homogenates from mice that had received rectal AdvmuIL-10 was mean 26.5 (SEM 8.7) pg/mg 
compared with $13(6.5) \mathrm{pg} / \mathrm{mg}$ in mice that had received an intravenous injection. In contrast, while liver and spleen IL-10 concentrations after rectal AdvmuIL-10 administration did not differ significantly from the background seen in PBS treated controls (the sensitivity of the ELISA was approximately 8 $\mathrm{pg} / \mathrm{mg})$, IL-10 concentrations in the liver and spleen of mice given an intravenous injection were significantly elevated ( 52.5 (22.5) pg/ml and 5.7 (4.3) pg/ml, respectively) (fig 2).

\section{Local AdvmulL-10 treatment is therapeutic for established colitis in IL-10-/- mice}

IL-10-/- mice develop a spontaneous enterocolitis associated with weight loss, passage of mucous, rectal prolapse, and diarrhoea. It has been reported that daily injections of recombinant murine IL-10 prevent disease but are not sufficient to reverse established colitis. ${ }^{14}$ In order to determine the therapeutic efficacy of local AdvmuIL-10 delivery, 10 week old IL-10-/- mice with established colitis received a single rectal instillation of $5 \times 10^{8}$ PFU of AdvmuIL-10, Adv0, or PBS vehicle. Wild-type mice received the same treatment to act as a negative control. IL-10-/- mice that received AdvmuIL-10 demonstrated a significant improvement in their colitis scores over the four week experiment whereas those that had received Adv0 or vehicle continued to develop progressive disease (fig 3). Thus the mean (SEM) clinical score in the AdvmuIL-10 group fell from $1.8(0.13)$ to $0.4(0.16)$ whereas clinical scores increased from $1.4(0.27)$ to $2.5(0.27)$ and from $1.8(0.22)$ to $2.6(0.13)$ in the PBS and Adv0 treated groups, respectively $(p<0.001$ for both Adv0 and PBS compared with AdvmuIL-10; two way ANOVA). No clinical evidence of colitis was detected in any wild-type mice, irrespective of their treatment group (data not shown). Analysis of weekly stool samples demonstrated that the progressive colitis observed in the Adv0 and PBS treated IL-10-/- mice was associated with release of significantly higher levels of the proinflammatory cytokine IL- $1 \beta$ than AdvmuIL-10 treated IL- $10-/-$ mice $(\mathrm{p}<0.01$; fig 3$)$. IL- $1 \beta$ was not detected in stools collected from wild-type mice treated with Adv0, AdvmuIL- 10, or PBS vehicle (data not shown).

Histological analysis of colonic tissue from 10 week old IL-10-/- mice demonstrated a marked colitis with a histological score of $9.4(1.3)(n=5)$. The histological score of colitic IL$10-/-$ mice four weeks after local instillation of $5 \times 10^{8} \mathrm{PFU}$ of Adv0 or saline vehicle increased to 11.1 (1.1) and 10.9 (1.0), respectively. The histological severity of colitis was consistent throughout the colon (data not shown). In contrast, a single dose of rectal AdvmuIL-10 induced histological remission in IL-10-/- mice with established disease (fig 4). Thus four weeks after AdvmuIL-10 therapy the mean histological score had fallen to 4.4 ( 1.5$)(\mathrm{p}<0.01$ compared with saline, $\mathrm{p}<0.001$ compared with Adv0 therapy); this was not significantly different from the scores of age matched wild-type mice that had received a rectal enema of saline, Adv0, or AdvmuIL-10 four weeks previously $(1.2(0.5), 3.2(0.8)$, and $1.4(0.6)$, respectively). Interestingly, local AdvmuIL-10 therapy ameliorated histological colitis throughout the colon as there were no significant differences between the histological scores of the proximal and distal sections of the colon in AdvmuIL-10 treated mice (data not shown).

\section{Local AdvmulL-10 treatment has no effect on TNF- $\alpha$ release from LPS stimulated splenocytes.}

IL-10-/- mice challenged with LPS released elevated levels of proinflammatory cytokines and suffered higher mortality than wild-type controls. ${ }^{20}$ We have reported that a single systemic injection of AdvmuIL-10 is sufficient to inhibit the elevated TNF- $\alpha$ and IFN- $\gamma$ release from LPS stimulated splenocytes harvested 10 weeks after therapy. ${ }^{13}$ In order to determine the systemic effects of local AdvmuIL-10 therapy, we compared TNF- $\alpha$ release from LPS stimulated spleen cells harvested from mice used in the above experiments. TNF- $\alpha$

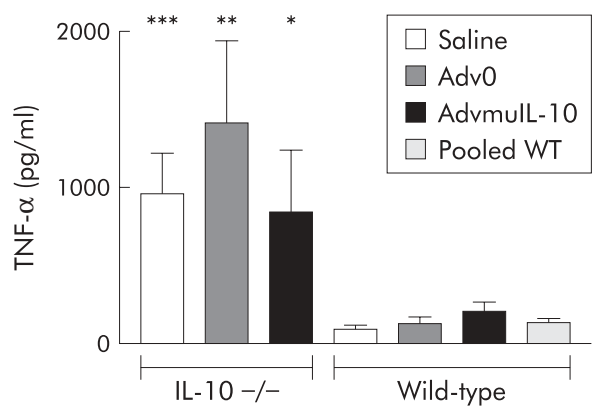

Figure 5 Rectal adenoviral vector encoding murine interleukin 10 (AdvmulL-10) did not diminish the lipopolysaccharide (LPS) splenocyte response in IL-10-/- mice. Splenocytes were isolated at sacrifice from both IL-10-/- ( $=10 /$ group) and wild-type ( $n=5$ /group) mice treated with $5 \times 10^{8}$ PFU empty cassette adenoviral vector (AdvO), saline, or AdvmulL-10 by rectal instillation four weeks previously. Cells $\left(2 \times 10^{6}\right.$ per well) were cultured for 24 hours in the presence of LPS $10 \mathrm{\mu g} / \mathrm{ml}$. Supernatants were harvested and assayed for tumour necrosis factor $\alpha$ (TNF- $\alpha$ ) by ELISA. Cells from each animal were assayed in triplicate; results are expressed as mean (SEM) for each group. ${ }^{\star} p \leqslant 0.05 ;{ }^{*} p \leqslant 0.01,{ }^{* *}{ }^{*} p<0.001$ versus pooled wild-type (WT) mice.

release from unstimulated splenocytes was below the sensitivity of the ELISA for all animals $(<40 \mathrm{pg} / \mathrm{ml})$. As expected, LPS $(10 \mu \mathrm{g} / \mathrm{ml})$ induced significantly higher levels of TNF- $\alpha$ from splenocytes harvested from IL-10-/- mice than wild-type controls (964 (266) pg/ml and 98 (21) pg/ml, respectively; $\mathrm{p}<0.02$, two tailed $t$ test). However, in contrast with systemic delivery, prior treatment of IL-10-/- mice with rectal AdvmuIL-10 had no effect on TNF- $\alpha$ release (fig 5). Thus TNF- $\alpha$ release from LPS stimulated splenocytes harvested from IL- 10-/- mice that had received rectal Adv0, AdvmuIL10 , or saline four weeks previously was $1414(530) \mathrm{pg} / \mathrm{ml}, 845$ (399) pg/ml, and 964 (266) pg/ml, respectively. Furthermore, neutralising IL-10 in LPS stimulated splenocyte cultures of IL-10-/- mice that had been treated with rectal AdvmuIL-10 had no effect on levels of TNF- $\alpha$ secreted (1326 (792) pg/ml with OX 20 isotype control compared with 1406 (676) pg/ml when cultured with JES52A5 anti-IL-10 antibodies). As a positive control for this experiment, TNF- $\alpha$ release from LPS stimulated splenocytes harvested from saline treated wildtype mice cultured with either JES52A5 or the OX20 was examined. As predicted, neutralising endogenous IL-10 significantly increased TNF- $\alpha$ release $(353(60) \mathrm{pg} / \mathrm{ml}$ with JES52A5 compared with 138 (26) pg/ml with OX20; p <0.02 by two tailed $t$ test).

\section{Neutralising antiadenovirus antibody response is diminished in AdvmulL-10 treated IL-10-/- mice}

Previous studies have shown elevated titres of antiadenovirus antibodies in mice treated with adenoviral vectors. ${ }^{21}$ We hypothesised that the neutralising antibody response to AdvmuIL-10 vectors would be diminished, as the protein encoded for by this virus will act to suppress both T cell activation and antigen presentation. A bioassay was used to detect the presence of antibodies directed to adenoviral proteins in the serum of mice that had received a rectal infusion of Adv0, AdvmuIL-10, or saline vehicle four weeks previously. Serum from both IL-10-/- and wild-type mice that had received rectal Adv0 contained neutralising antiadenoviral antibodies were compared with serum from mice that had received saline $(p<0.001$ two way ANOVA; fig 6). However, there was no difference in the antiadenoviral antibody titre in serum from IL-10-/- mice that had received rectal AdvmuIL-10 compared with those that had received saline. Interestingly, the diminished antiadenoviral response to AdvmuIL-10 was less pronounced in wild-type mice than in IL-10-/- mice. 

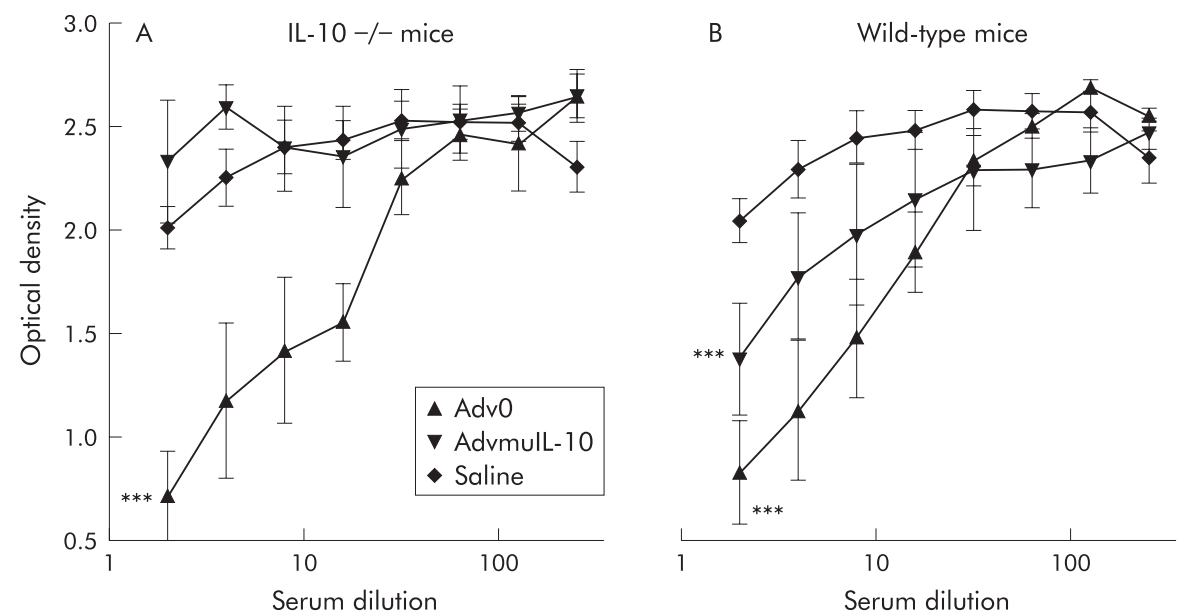

Figure 6 Rectal adenoviral vector encoding murine interleukin 10 (AdvmulL-10) induced a diminished antiadenoviral response in IL-10-/mice. The neutralising antiadenovirus antibody titre was analysed in serum from (A) IL-10-/- and (B) wild-type mice four weeks after rectal instillation of $5 \times 10^{8} \mathrm{PFU}$ of AdvmulL-10, empty cassette adenoviral vector (Adv0), or saline vehicle. Serial serum dilutions were incubated for 90 minutes at $37^{\circ} \mathrm{C}$ with $2 \times 10^{\circ} \mathrm{PFU}$ of adenoviral vector encoding $\beta$-galactosidase (Adv $\beta$ gal) and applied in duplicate to $80 \%$ confluent 293 cells on a 96 well plate. After one hour at $37^{\circ} \mathrm{C}, 50 \mu$ of Dulbecco's modified Eagle's medium containing $10 \%$ fetal calf serum was added to each well, and cells were cultured for a further 36 hours. Cell supernatants were then removed and assayed using a $\beta$-galactosidase substrate solution. In this assay, a low optical density reflects a high titre of serum antiadenoviral antibodies. Results are expressed as mean (SEM) optical density for each group. ${ }^{* *} p<0.001$ compared with saline treated mice, two way ANOVA).

\section{DISCUSSION}

We have explored the potential of local AdvmuIL-10 administration as a therapy for intestinal inflammation. This strategy has the advantage that IL-10 delivery is targeted to the sites of inflammation which may prevent the side effects associated with high dose systemic IL-10 administration. The use of the IL-10-/- model of colitis allows accurate determination of the site of IL-10 expression after local AdvmuIL-10 delivery. In addition, as daily systemic injections of murine IL-10 are unable to reverse established colitis in this model, ${ }^{14}$ it allows a comparison of the therapeutic efficacy of local AdvmuIL-10 and recombinant IL-10 injections.

Adenoviral vectors bind to cell surface integrins $(\alpha v \beta 3$ and $\alpha v \beta 5$ ) and gain entry by receptor mediated endocytosis using a receptor such as the Coxsackie virus and adenovirus receptor (CAR) ${ }^{22}$ CAR mRNA can be detected in human intestinal tissue by northern blotting. ${ }^{23}$ Thus infection of human colonic epithelial cells with AdvmuIL-10 in vitro resulted in the release of high levels of functional IL-10 as determined by a specific bioassay. The effect of the secreted murine IL-10 on epithelial cell function was not assessed, as despite $73 \%$ amino acid homology between murine and human IL-10 proteins, murine IL-10 has no activity on human cells. ${ }^{24}$

The presence of CAR on murine colonic epithelial cells has not been determined. ${ }^{23}$ However, recent reports have suggested that the MHC class I receptor may also function as a high affinity receptor for adenoviral vectors. ${ }^{25}$ Irrespective of this, previous studies in mice have demonstrated that colonic administration of adenoviral vectors leads to colonic reporter gene expression, which peaks at $48-72$ hours. ${ }^{16}{ }^{19}$ These findings were confirmed by the significant levels of IL-10 that were detected in colonic homogenates of IL-10-/- mice one week after local AdvmuIL-10 administration. The duration of IL-10 expression in this experiment is surprising considering that colonic epithelial cell turnover occurs every 2-3 days. ${ }^{26}$ However, Foreman et al also reported low level $\beta$-galactosidase expression for up to 180 hours after colonic Advßgal administration. ${ }^{19}$ Although rectal administration of adenoviral vectors predominantly targets the colonic epithelial cell, some expression has been reported in laminn propria mononuclear (LPMN) cells. ${ }^{16}$ Thus the late phase of gene expression after local adenoviral vector administration may represent LPMN cell infection. Furthermore, Wirtz et al have reported that the ratio of LPMN cells to epithelial cells infected by rectal adenoviral administration is increased in mice that have colitis. ${ }^{16}$ This may explain the prolonged duration of IL-10 expression that we report after AdvmuIL-10 delivery to IL-10-/- mice with established colitis. In addition, AdvmuIL-10 induces a diminished host antiadenovirus response in IL-10-/mice compared with control vectors that may also permit prolonged IL-10 expression.

Whereas systemic AdvmuIL-10 administration leads to IL-10 expression in the liver, spleen, and colon, IL-10 protein was not detected in the liver and spleen of IL-10-/- mice that received AdvmuIL-10 as an enema. The absence of systemic IL-10 activity after local AdvmuIL-10 therapy was confirmed by experiments examining the response of harvested splenocytes to stimulation with LPS. Local delivery of replication deficient adenoviral vectors was well tolerated and did not exacerbate intestinal inflammation. Studies of adenoviral vectors encoding non-immunoregulatory proteins have demonstrated host antivirus immune responses that limit the duration of gene expression and prevent retreatment. ${ }^{21}$ However, in contrast with the marked antibody response to control vectors, local AdvmuIL-10 delivery significantly diminished the host antiadenoviral response in IL-10-/- mice. It is interesting that the reduction in antiadenoviral response seen with AdvmuIL-10 administration is not as marked in wild-type as in IL-10-/- mice. The explanation for this dichotomy is not clear but is likely to represent alterations in B cell maturation in IL-10-/- mice. ${ }^{4}$

IL-10-/- mice develop a spontaneous Th-1 cell mediated enterocolitis with many similarities to Crohn's disease. ${ }^{4}$ Steidler et al investigated the therapeutic efficacy of local IL-10 delivery using bacteria that had been genetically modified to secrete high levels of IL- $10 .{ }^{15} \mathrm{~A}$ four week oral course of these bacteria led to a $75 \%$ reduction in the histological severity of colitis in IL-10-/- mice. However, this therapy was administered to three week old mice prior to the onset of their disease. In contrast, we demonstrated that, unlike daily IL- 10 injections, a single AdvmuIL-10 enema was capable of reversing established disease in IL- 10-/- mice. Furthermore, clinical remission was maintained for at least four weeks after therapy. The dramatic improvement in clinical score was associated with a fall in the levels of the proinflammatory cytokine IL- $1 \beta$ that was detected in high concentrations in the stool of IL-10-/- mice with colitis. Most strikingly, rectal AdvmuIL-10 therapy led to a $60 \%$ reduction in the histological severity of 
colitis compared with control treated mice. Thus a single dose of IL-10 gene therapy delivered directly to the colon was sufficient to suppress established disease in IL-10-/- mice for at least four weeks.

The mechanisms by which IL-10 induced by AdvmuIL-10 therapy could have diminished disease severity in the intestine were not addressed directly in our experiments. However, they include downregulation of antigen presenting cell activity and inhibition of proinflammatory cytokine production. The fact that the therapeutic effects of AdvmuIL-10 therapy persisted for at least four weeks and extended throughout the colon suggest that its mechanism of action may extend beyond the local suppression of proinflammatory cytokine release. An analogous situation occurs in mice with collagen induced arthritis in which an intrarticular injection of AdvmuIL-10 is therapeutic for both the treated and contralateral paw. ${ }^{27}$ As in our experiments, the duration of this therapeutic effect persisted far longer than the period in which local IL-10 can be detected. Thus it is possible that local IL-10 release induces differentiation or activation of regulatory $\mathrm{T}$ cell clones. ${ }^{7}$ This hypothesis is supported by studies of transgenic mice that overexpress $\mathrm{IL}-10$ in the intestinal epithelium. ${ }^{28}$ The mucosal lymphocyte population of these transgenic mice contains a higher proportion of immunoregulatory $\mathrm{CD} 4^{+} \mathrm{CD} 25^{+} \mathrm{T}$ cells than wild-type controls. Furthermore, stimulated intestinal lymphocytes from IL-10 transgenic mice secrete lower levels of Thl cytokines and higher levels of transforming growth factor $\beta$ than controls. ${ }^{28}$

Thus delivery of AdvmuIL-10 directly to the intestinal lumen provides local IL-10 production minimising the side effects associated with systemic therapy. Most strikingly, local AdvmuIL-10 therapy induces clinical and histological remission in IL-10-/- mice with established colitis. The concept of local gene delivery as a therapy for intestinal inflammation is supported by the demonstration that intrarectal administration of an El deleted adenoviral vector expressing IL-18 antisense mRNA inhibits mucosal IFN- $\gamma$ release and suppresses inflammation in the transfer model of colitis. ${ }^{29}$ However patients with Crohn's disease are not IL-10 deficient and may mount an immune response to AdvmuIL-10, as seen in wildtype mice. This would limit the duration of a therapeutic response and prevent retreatment. Alternative viral vectors may be more appropriate for clinical trials of IL-10 gene therapy than the replication deficient adenoviral vectors used in the current study. Adeno associated viruses are less immunogenic, and by integrating into the host genome have been shown to provide long term gene expression in the intestinal tract. ${ }^{30}$ Furthermore, therapy could be targeted to sites of active disease using an inflammation inducible C3-tat/HIV promoter that induces IL-10 release in response to inflammatory stimuli such as TNF- $\alpha$. $^{31}$

In conclusion, local gene therapy strategies using viral vectors encoding immunoregulatory cytokines may prove to be a potent approach to the treatment of chronic inflammatory diseases such as Crohn's disease.

\section{ACKNOWLEDGEMENTS}

We gratefully acknowledge Patricia Green for her technical assistance, and Philip Connolly who kindly performed the histological processing. This work was supported by the Digestive Diseases Foundation and a Clinical Training Fellowship from the Medical Research Council of the UK.

\section{Authors' affiliations}

J O Lindsay, C J Ciesielski, F M Brennan, Kennedy Institute of Rheumatology Division, Imperial College School of Medicine, London, UK T Scheinin, Fourth Department of Surgery, University of Helsinki Central Hospital, Kasarmikatu 11-13, FIN-00130 Helsinki, Finland H J Hodgson, Department of Hepatology, Royal Free and University College Medical School, Royal Free Campus, London, UK

\section{REFERENCES}

1 Duchmann R, Kaiser I, Hermann E, et al. Tolerance exists towards resident intestinal flora but is broken in active inflammatory bowel disease (IBD). Clin Exp Immunol 1995;102:448-55.

2 Pallone F, Fais S, Squarcia O, et al. Activation of peripheral blood and intestinal lamina propria lymphocytes in Crohn's disease. In vivo state of activation and in vitro response to stimulation as defined by the expression of early activation antigens. Gut 1987;28:745-53.

3 Fiocchi C. Inflammatory bowel disease: etiology and pathogenesis. Gastroenterology 1998;115:182-205.

4 Kuhn R, Lohler J, Rennick D, et al. Interleukin-10-deficient mice develop chronic enterocolitis. Cell 1993;75:263-74.

5 Spencer SD, Di Marco F, Hooley J, et al. The orphan receptor CRF2-4 is an essential subunit of the interleukin 10 receptor. J Exp Med 1998; 187:571-8

6 Moore KW, de Waal Malefyt R, Coffman RL, et al. Interleukin-10 and the interleukin-10 receptor. Annu Rev Immunol 2001; 19:683-765.

7 Groux H, O'Garra A, Bigler M, et al. A CD4+ T-cell subset inhibits antigen-specific T-cell responses and prevents colitis. Nature 1997;389:737-42.

8 Tomoyose M, Mitsuyama K, Ishida H, et al. Role of interleukin- 10 in a murine model of dextran sulfate sodium-induced colitis. Scand J Gastroenterol 1998;33:435-40.

9 Powrie F, Leach MW, Mauze S, et al. Inhibition of Th 1 responses prevents inflammatory bowel disease in scid mice reconstituted with CD45RBhi CD4+ T cells. Immunity 1994:1:553-62.

10 Schreiber S, Heinig T, Thiele HG et al. Immunoregulatory role of interleukin 10 in patients with inflammatory bowel disease. Gastroenterology 1995; 108:1434-44.

11 Schreiber S, Fedorak RN, Nielsen $\mathrm{OH}$, et al. Safety and efficacy of recombinant human interleukin 10 in chronic active Crohn's disease. Gastroenterology 2000;1 19:1461-72.

12 Fedorak RN, Gangl A, Elson CO, et al. Recombinant human interleukin 10 in the treatment of patients with mild to moderately active Crohn's disease. Gastroenterology 2000;1 19:1473-82.

13 Lindsay JO, Ciesielski CJ, Scheinin T, et al. The prevention and treatment of murine colitis using gene therapy with adenoviral vectors encoding IL-10. J Immunol 2001;166:7625-33.

14 Berg DJ, Davidson N, Kuhn R, et al. Enterocolitis and colon cancer in interleukin-10- deficient mice are associated with aberrant cytokine production and CD4(+) TH1-like responses. J Clin Invest production and CD4

5 Steidler L, Hans W, Schotte L, et al. Treatment of murine colitis by Lactococcus lactis secreting interleukin-10. Science 2000;289:1352-5.

16 Wirtz S, Galle PR, Neurath MF. Efficient gene delivery to the inflamed colon by local administration of recombinant adenoviruses with normal or modified fibre structure. Gut 1999;44:800-7

17 Graham FL, Prevec L. Methods for construction of adenovirus vectors. Mol Biotechnol 1995;3:207-20.

18 David A, Coupel-Clauce H, Chetritt J, et al. Anti-adenovirus immune responses in rats are enhanced by interleukin 4 but not interleukin 10 produced by recombinant adenovirus. Hum Gene Ther 1998:9: 1755-68

19 Foreman PK, Wainwright $\mathrm{W}$, Alicke B, et al. Adenovirus-mediated transduction of intestinal cells in vivo. Hum Gene Ther 1998;9:1313-21.

20 Berg DJ, Kuhn R, Rajewsky K, et al. Interleukin-10 is a central regulator of the response to LPS in murine models of endotoxic shock and the Shwartzman reaction but not endotoxin tolerance. J Clin Invest 1995; $96: 2339-47$

21 Yang $Y, L i Q, E r t l ~ H C$, et al. Cellular and humoral immune responses to viral antigens create barriers to lung-directed gene therapy with recombinant adenoviruses. J Virol 1995;69:2004-15.

22 Bergelson JM, Cunningham JA, Droguett $G$, et al. Isolation of a common receptor for Coxsackie B viruses and adenoviruses 2 and 5 . Science 1997;275:1320-3.

23 Tomko RP, Xu R, Philipson L. HCAR and MCAR: the human and mouse cellular receptors for subgroup $C$ adenoviruses and group $B$ coxsackieviruses. Proc Natl Acad Sci U S A 1997;94:3352-6.

24 Moore KW, O'Garra A, de Waal Malefyt R, et al. Interleukin-10. Annu Rev Immunol 1993; 11:165-90.

25 Hong SS, Karayan L, Tournier J, et al. Adenovirus type 5 fiber knob binds to $M H C$ class I alpha2 domain at the surface of human epithelial and B lymphoblastoid cells. Embo J 1997:16:2294-306.

26 Gordon JI, Schmidt GH, Roth KA. Studies of intestinal stem cells using normal, chimeric, and transgenic mice. Faseb J 1992;6:3039-50.

27 Whalen JD, Lechman EL, Carlos CA, et al. Adenoviral transfer of the viral IL-10 gene periarticularly to mouse paws suppresses development of collagen-induced arthritis in both injected and uninjected paws. J Immunol 1999:162:3625-32.

28 De Winter H, Elewaut D, Turovskaya O, et al. Regulation of mucosal immune responses by recombinant interleukin 10 produced by intestinal epithelial cells in mice. Gastroenterology 2002;122:1829-41.

29 Wirtz S, Becker C, Blumberg R, et al. Treatment of T cell-dependent experimental colitis in SCID mice by local administration of an adenovirus expressing IL-18 antisense mRNA. J Immunol 2002:168:411-20

30 During MJ, Xu R, Young D, et al. Peroral therapy of lactose intolerance using an adeno-associated virus vector. Nat Med 1998;4:1131-5.

31 Miagkov AV, Varley AW, Munford RS, et al. Endogenous regulation of a therapeutic transgene restores homeostasis in arthritic joints. J Clin Invest 2002;109:1223-9. 